\title{
PREDICTING THE BREAKING STRENGTH OF GRAVITY WATER WAVES FROM DEEP TO SHALLOW WATER
}

\author{
James T. Kirby, University of Delaware, kirby@udel.edu \\ Morteza Derakhti, Johns Hopkins University, derakhti@jhu.edu \\ Michael L. Banner, University of New South Wales, m.banner@unsw.edu.au
}

\section{PROBLEM STATEMENT}

We revisit the classical but as yet unresolved problem of predicting the breaking strength of 2-D and 3-D gravity water waves. The most commonly used approach is the Phillips/Duncan spectral framework for the mean breaking crest length per unit area, combined with a scaling argument for the wave energy dissipation rate per unit length of breaking crest, giving a total dissipation written as

$$
\epsilon=b \rho g^{-1} c_{b}^{5}
$$

where $\varepsilon$ is the total wave energy dissipation due to wave breaking, $\rho$ is the density of the liquid phase, $g$ is the gravitational acceleration, $c_{b}$ is the phase speed of the breaking wave, and $b$ is the dimensionless breaking strength parameter.

Previous efforts to develop parameterizations of $b$ based on laboratory and field data usually employ a measure based on spectral wave steepness, as reviewed by Romero et al. (2012) and Derakhti \& Kirby (2016). These measures are difficult to construct in the absence of full knowledge of the wave field, and are likewise not well constrained by properties of the local wave during a breaking event. Finally, previous studies have reported values of $b$ for breaking events in modulated wave trains which fall appreciably below values reported for singlebreaking focused wave packets, suggesting a significant physical difference between the mechanism of breaking in the two types of events. This can be primarily attributed to different choices for averaging periods, as discussed in detail in Derakhti et al. (2018).

Barthelemy et al. (2018) showed that highest nonbreaking waves were clearly separated from marginally breaking waves by their normalized energy fluxes localized near the crest tip region, and that initial breaking instability occurs within a very compact region centered on the wave crest. On the surface, the expression for normalized energy flux (denoted by symbol $B$ ) reduces to the ratio of fluid velocity at the crest to the translational velocity of the crest for the tallest wave in the evolving group. Barthelemy et al. (2018) found that a value of $B_{t h}=0.85$ provides a robust threshold for breaking onset for 2-D wave packets propagating in deep or intermediate uniform water depths. Further targeted study of representative cases of the most severe laterally-focused 3-D wave packets in deep and intermediate depth water shows that the threshold remains robust. These numerical findings for 2-D and 3-D cases have since been supported by laboratory observations.
Our goal is to find a robust and local parameterization to predict the breaking strength of 2-D and 3-D gravity water waves. We use a LES/VOF model described by Derakhti \& Kirby (2014) to simulate nonlinear wave evolution, breaking onset and post-breaking behavior for representative cases of focused wave packets or modulated wave trains. Using these numerical results, we investigate the relationship between the breaking strength parameter $b$ and the breaking onset parameter $B$ proposed by Barthelemy et al. (2018). While the results are potentially applicable more generally, in this paper we concentrate on breaking events due to focusing or modulational instability in wave packets over flat bottom topography and for conditions ranging from deep to intermediate depth, with depth to wavelength ratios ranging from 0.68 to 0.13 .

\section{NUMERICAL EXPERIMENTS}

A detailed description of the polydisperse two-fluid model and boundary conditions used may be found in Derakhti \& Kirby (2014). Numerical experiments conducted here use formulations for 2D (Derakhti \& Kirby, 2014\} and 3D (Kirby \& Derakhti, 2017) focused wave packets, or by Banner \& Peirson (2007) for modulated wave trains. Complete details may be found in Derakhti et al. (2018). The coordinate system $(x, y, z)$ is defined with $x$ and $y$ representing the along-tank and transverse directions and $z$ is the vertical direction, positive upward and measured from the still water level. The reference time $t^{\wedge *}$ and location $\mathrm{x}^{\wedge *}$ are taken as the time and location at which $B$ following the crest tip reaches the threshold value of 0.85 for breaking packets, or its maximum for nonbreaking packets, and are normalized by the local period and wave length of the carrier wave. Incident waves are generated at the model upstream boundary. Finally, fluid velocities for each component are calculated using linear theory and then superimposed at the wavemaker. Table 1 in Derakhti et al. (2018) summarizes the input parameters for all simulated cases.

\section{PARAMETERIZATION OF BREAKING STRENGTH}

Figure 1 shows snapshots of free surface elevations before and after breaking onset as well as the temporal variation of $B$ for focused packets with weak spilling or strong plunging breakers. Results show that, as the strength of breaking increases, the rate of change $d B / d t$ near the threshold value $B_{t h}$ increases. Warning of imminent breaking onset $\left(\mathrm{t}^{\star}=0\right.$ here) occurs up to a fifth of a wave period prior to a breaking event. As a consequence, the wave form at $B=B_{t h}$ is well defined and the free surface is single-valued. Results for 

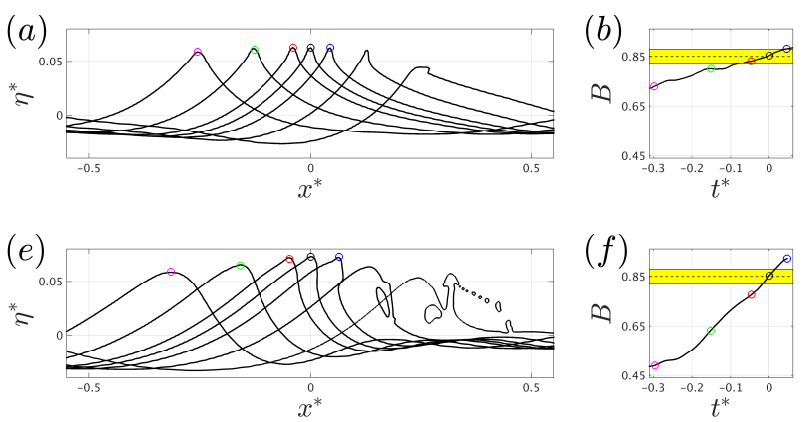

Figure 1. Snapshots of the spatial variation of free surface elevations near the maximum crest (left) and corresponding variation of $B$ following the crest maximum (right) for a weak spilling breaker (top) and strong plunging breaker (bottom).

breaking in packets focused in 3D are further illustrated in Derakhti et al. (2018) and are largely consistent with the $2 \mathrm{D}$ cases shown here.

To construct a non-dimensional parameter representing breaking events, we need to identify an appropriate time scale. We choose the local period, $T_{b}$, of the carrier wave at $B=B_{t h}$, obtained by using the linear dispersion relation and the local wave length $L_{b}$ defined based on the two successive zero up- and zero down-crossing points around the crest maximum. Figure 2 shows the variation of the breaking strength parameter $b$ with the new parameter $\Gamma=T_{b} d B / d t \mid B_{t h}$ for all simulated cases. The methodology for calculating $b$ from computed results is discussed in Derakhti et al. (2018). We found that model estimates of the loss of total wave energy due to wave breaking are typically within $10 \%$ of observed levels, after correcting for the change in the downstream group velocity following breaking. As a result, the uncertainty in the predicted $b$ values are expected to be less than $10 \%$ for the cases considered here. The results show that $\Gamma$ can successfully predict the breaking strength parameter $b$. The dashed curve in Figure 2 represents a leastsquare curve fit to the full set of results, and is given by

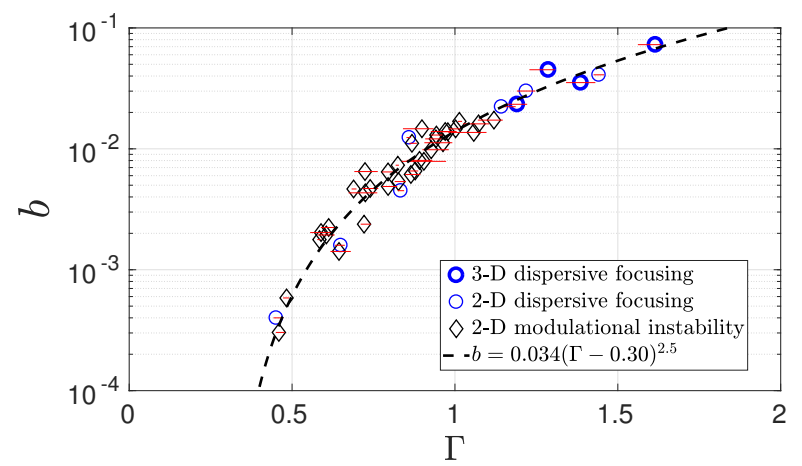

Figure 2. Variation in the breaking strength parameter $b$ with the new parameter $\Gamma=\mathrm{Tb} \mathrm{dB} / \mathrm{dt} \mid \mathrm{Bth}$, representing the normalized rate of change of $B$ as it transitions through the breaking onset. The dashed line indicates the fitted curve in equation (2).

$$
b=0.034(\Gamma-0.30)^{5 / 2}
$$

CONCLUSIONS AND ONGOING WORK

The results shown here show a systematic collapse of the proposed breaking strength predictor $\Gamma$ for a diverse range of representative $2 D$ and $3 D$ water wave trains. The results here, based on a local averaging over individual breaking wave crests, do not show a pronounced difference between values of $b$ obtained for focused packets or modulational events.

The success of the parameterization for predicting breaking strength found here would make it possible to better describe breaking events in codes based on potential flow theory, such as high-order spectral (HOS) codes, where breaking is not predicted by the model itself. The development of criteria for the onset and strength of breaking in such models has long been a subject for investigation. The specification of such a dissipation model should be based on the parameterization of total dissipation in terms of the rate of change parameter $\Gamma$ developed here, which would provide a strong link between the present work and operational wave modeling.

The present work provides a first indication of a direct link between the local properties of a wave crest as it transitions through an apparently generic breaking threshold, and the resultant overall energy dissipation resulting from the breaking event. The universality of the $B$ parameter and its rate of change as robust indicators of wave breaking onset and strength clearly warrant further study. The results presented here are presently being extended to cases involving depth-limited breaking and breaking during wave blocking on opposing currents.

Acknowledgments: This work was supported by the National Science Foundation, Physical Oceanography Program. M.B. acknowledges support from the Australian Research Council.

\section{REFERENCES}

Banner, Peirson (2007): Wave breaking onset and strength for two-dimensional deep-water wave groups. J. Fluid Mech., 585, 93-115.

Barthelemy, Banner, Peirson, Fedele, Allis, Dias (2018) On a unified breaking onset threshold for gravity waves in deep and intermediate depth water. J. Fluid Mech., 841, 463-488. Derakhti, Kirby (2014): Bubble entrainment and liquid bubble interaction under unsteady breaking waves, J. Fluid Mech., 761, 464-506.

Derakhti, Kirby (2016): Breaking-onset, energy and momentum flux in unsteady focused wave packets, J. Fluid Mech., 790, 553-581.

Derakhti, Banner, Kirby (2018): Predicting the breaking strength of gravity water waves in deep and intermediate depth, J. Fluid Mech., in press.

Kirby, Derakhti (2018): Short-crested wave breaking, European J. Mechanics/B Fluids, in press.

Romero, Melville, Kleiss (2012): Spectral energy dissipation due to surface wave breaking, J. Phys. Oceanogr., 42, 14211444. 